\title{
A MODIFIED ALgORITHM FOR THRESHOLDING AND DETECTION OF FACIAL INFORMATION FROM Color IMAges USING Color CENTROID SEgMENTATION AND CONTOURLET TRANSFORM
}

\author{
Sunish Kumar O S ${ }^{1}$ and Akash G Kamal ${ }^{2}$ \\ ${ }^{1}$ Asst. Professor, Department of Electronics and Communication Engineering, \\ Amal Jyothi College of Engineering, Kerala, India \\ ossunishkumareamal jyothi.ac. in \\ 2 Asst. Professor, Department of Electronics and Communication Engineering, \\ Amal Jyothi College of Engineering, Kerala, India \\ akasgkamal@amaljyothi.ac.in
}

\begin{abstract}
Human face detection plays an important role in many application areas such as video surveillance, human computer interface, face recognition, face search and face image database management etc. In human face detection applications, face region usually form an inconsequential part of images. Preliminary segmentation of images into regions that contain "non-face" objects and regions that may contain "face" can greatly accelerate the process of human face detection. This can be done using skin color segmentation, where given image is segmented based on color as 'skin region' and 'non skin regions'. Thus we can say that the skin regions may contain face and other regions don't. Color information based methods take a great attention, because colors have obviously character and robust visual cue for detection. This paper proposes a method based on RGB color centroids segmentation (CCS) for face detection. This paper includes two parts, first part is color image thresholding based on CCS to perform skin color segmentation and the then detection of human face from detected skin regions. CCS method has some shortcomings as it fails when the skin color of the subject lacks chroma. This happens especially with subjects having too darker or too lighter skin tones. This shortcoming of CCS can be overcome using Contourlet Transformation. In this paper, we pursue a two dimensional transform that can capture the intrinsic geometrical structure that is key in visual information.
\end{abstract}

\section{KEYWORDS}

Face Detection, Color Centroid Segmentation, Thresholding, Contourlet Transform, Skin Color Segmentation.

\section{INTRODUCTION}

Nowadays, many application technologies are developed to secure access control, are based on biometrics recognition such as fingerprints, iris pattern and face recognition[9]. Along with the development of those technologies, computer controller plays an important role to make the biometrics recognition more economically feasible in such developments. Face recognition is a major concerned research direction in this field. In recent years, the face recognition become popular research direction more and more, and has many applications such as financial transactions, monitoring system, credit card verification, ATM access, personal PC access, video surveillance etc. There are numerous researches going on in this field all over the world. Face detection plays an important role in today's world. However research in this field is still

DOI : $10.5121 /$ sipij.2011.2212 
Signal \& Image Processing : An International Journal (SIPIJ) Vol.2, No.2, June 2011

young. Face recognition depends heavily on the particular choice of features used by the classifier. One usually starts with a given set of features and then attempts to derive an optimal subset (under some criteria) of features leading to high classification performance with the expectation that similar performance can also be displayed on future trials using novel (unseen) test data.All of the research studies find its basics in CBIR [5], [6], [8], [10]. Content-based image retrieval (CBIR), also known as query by image content (QBIC) and content-based visual information retrieval (CBVIR) is the application of computer vision to the image retrieval problem, that is, the problem of searching for digital images in large databases.

"Content-based" means that the search will analyze the actual contents of the image. The term 'content' in this context might refer colours, shapes, textures, or any other information that can be derived from the image itself. Without the ability to examine image content, searches must rely on metadata such as captions or keywords, which may be laborious or expensive to produce.

This paper proposes a new method of face detection based on Color Centroids Segmentation (CCS) [1] and Contourlet Transformation (CT) [7].This method is able to handle a wide range of variations in color image sequence, various backgrounds, various lighting conditions and various skin tones for detection of face region efficiently. The rest of this paper is organized as follows. Section II describes how to create CCS model and use it to thresholding. Section III describes about thresholding based on CT model. Section IV uses proposed CCS model to detect face region. Section V presents the experimental results. Section VI gives the conclusions and the problems for future works.

\section{Color IMAge Thresholding BaSed ON CCS}

This section introduce how to thresholding the color image by $\mathrm{CBH}$ model though analyzing created color triangle and its color centroids region distribution. Here describes how to transform RGB components of RGB 3-D color space to 2-D polar coordinate system, and use multithreshold to segment the centroids region. By analyzing and processing, it can cluster the color of image to 2 7 colors by 2 7 thresholds for require and the effect better than traditional methods.

\subsection{Color Triangle}

In image processing, RGB, YCbCr, HSV, HSI etc. color spaces are widely used. These color spaces use three components to reflect color information, e.g. RGB color space consist of $R, G$ and $B$ components. This paper tries to transform the 3-D color space to 2-D coordinate system by color triangle (Fig. 1). To create the color triangle, a standard 2-D Cartesian coordinate system is used to describe $R, G$ and $B$ values and then transform it to polar coordinate system as Shown in figure (1):

$$
\begin{aligned}
& \operatorname{R}: r\left(\varphi_{R}\right)=r_{R},\left(\varphi_{R}=90^{0} \& 0 \leq r_{R} \leq 255\right) \\
& G: r\left(\varphi_{G}\right)=r_{G},\left(\varphi_{G}=210^{0} \& 0 \leq r_{G} \leq 255\right) \\
& B: r\left(\varphi_{B}\right)=r_{B},\left(\varphi_{B}=330^{\circ} \& 0 \leq r_{B} \leq 255\right)
\end{aligned}
$$

By the following steps can create the color triangle:

Step 1: create a standard 2-D polar coordinate system;

Step 2: create three color vectors to reflect $R, G$ and $B$ colors; every vector's value range is [0, 255 ] and alternation $120^{\circ}$ reciprocally.

Step 3: connect the three apexes. After above processes, the color triangle can be created as Fig. 1. For different $R, G$ and $B$ values, the shape of triangle is changeable. No matter the $R, G$ and $B$ value change the main structure are fixed. 


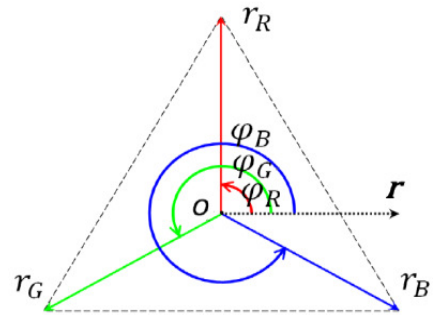

Fig. 1 RGB color triangle

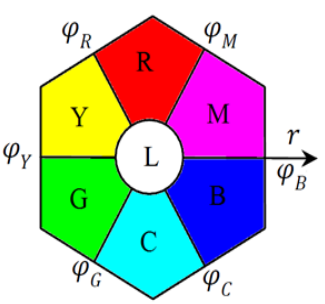

Fig. 2 CCS model

\subsection{Color Centroids Hexagon Region Distributing}

Because of R, G, B vectors direction is fixed and the value is change from 0 to 255 , so different combination of R, G, B value will create different color; also, the shape of color triangle is changed too. The different shape triangle has different centroid, and the centroids distributing region of color triangle is show hexagon as Fig. 2. In this hexagon region, it divided to 7 regions: R (Red), G (Green), B (Blue), C (Cyan), M (Magenta), Y (Yellow) and L (Luminance, achromatic) regions. In Fig. 2 we use seven threshold curves as the dividing line for thresholding. By observing the relation of color and corresponding centroid position of color triangle, we find that if the $R, G$ and $B$ values are closely, no matter small or large it only reflect the luminance information (weak color information). So the centroids of corresponding color triangles will in a circular region ( $\mathrm{L}$ region). And other six color regions reflect the color character of $\mathrm{R}, \mathrm{G}$ and $\mathrm{B}$ combination.

\subsection{Color Centroids Segmentation Thresholds Acquisition for skin color segmentation}

Considering the L region usually is not the goal region and existing method is not efficient to divide white and black region usually. This region is noise region, so clustering the value of this kind to one region wills effective to ignore the influence of white, black and other achromatic region. Here let $r_{\mathrm{L}}$ as the threshold of $\mathrm{L}$ region, $\varphi$ as the angle, the function of threshold curve is:

$\mathrm{r}(\varphi)=\mathrm{r}_{\mathrm{L}}\left(0^{\circ} \leq \varphi \leq 360^{\circ}\right)$

By observing the distributing of color centroids in hexagon region as Fig. 3(b), it can be seen that the centroid distributing of different color are different. Only when the $R=G=B$, the centroid is the origin of hexagon region. The color information is stronger proportional to the distance from origin. For example, $(R, G, B)=(255,0,0)$ reflect the red color and the centroid is in the upper peak of Fig. 2. By analyzing centroid distribution of face region, we can see that the color of face usually include Red region and lean to Yellow region. Thus we can choose $\varphi_{\mathrm{R}}$ and $\varphi_{\mathrm{Y}}$ as thresholds for clustering the image to face region and non-face region. But using fixed value for thresholding cannot give ideal thresholding result for different image conditions such as varying skin tone, white balance of image, lighting conditions etc. Thus, here proposes an automatic threshold acquisition method to acquire the thresholds for any given image. But finding the segmentation borderline of the colors in Fig. 3(c) is not easy. To display distributing character of centroids more clearly, we transform the Polar coordinate system to Cartesian coordinate system as Fig. 3(c) to reflect the distribution of centroids. In the Fig. 3(c) horizontal axis is $\varphi\left(\varphi \square\left(0^{\circ}\right.\right.$, $\left.360^{\circ}\right]$ ), vertical axis shows the percentage of regions of image having the given angle, $\varphi$ and other six vertical color-line are color threshold curves $\left(\varphi_{\mathrm{M}}, \varphi_{\mathrm{R}}, \varphi_{\mathrm{Y}}, \varphi_{\mathrm{G}}, \varphi_{\mathrm{C}}\right.$ and $\left.\varphi_{\mathrm{B}}\right)$. The face region is belonging to Red and Yellow region ( $\varphi_{\text {face }} \square\left[\varphi_{\mathrm{M}}, \varphi_{\mathrm{Y}}\right]$ ) 


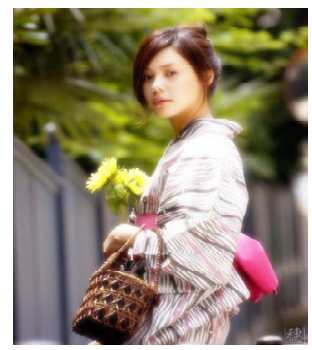

(a) Original image

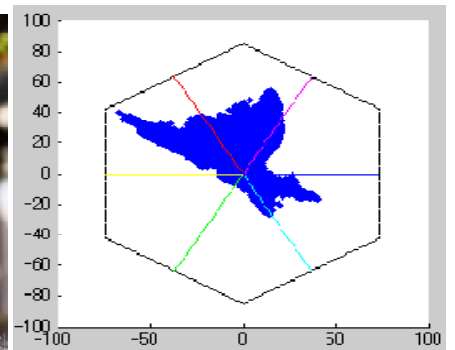

(b) Color centroids distributing

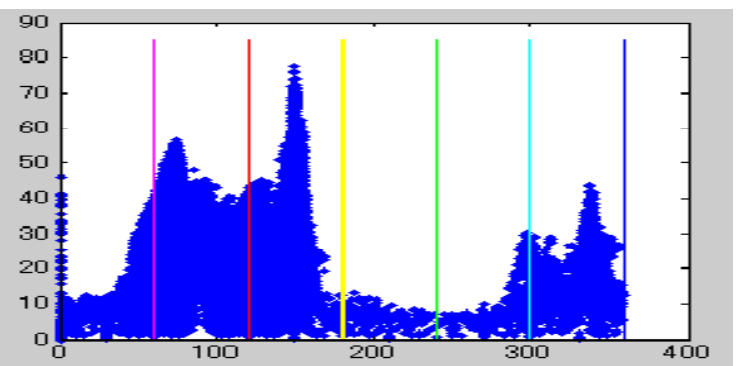

(c) Color centroids distributing conversion and thresholds selection

Figure 3.

Color centroids distributing and selection of sample image 1 has been shown in the above figure. For segmentation of face region, it must calculate the accurate value. Because of the distributing histogram is not smooth, we use filter to make the smooth shape for analyzing. By observing many faces, including images in different conditions, we let the threshold curve $\varphi_{M}$ and $\varphi_{Y}$ can move to left or right by $20^{\circ}$ for finding the best value. Then find the left and right valley bottom respectively as $\varphi^{\prime}{ }_{M}$ and $\varphi_{Y}^{\prime}$ in the fix range by histogram analysis method. For calculating the $\mathrm{r}_{\mathrm{L}}$ we fix the range from 3 to 20, and calculate the average value of every valley bottom (on color region only calculate one minimum value). In Fig. 3, (a) is original image and (b) showing the distribution of color centroids. By transforming Fig. 3(b) to (d) and calculate the $\varphi^{\prime}{ }_{M}, \varphi_{Y}^{\prime}$ and $\mathrm{T}_{\mathrm{L}}$, we can get the pre-face region and the binary image show in Fig. 3(c).

$$
\mathrm{r}(\varphi)=\mathrm{r}_{\text {face }} ;\left(\varphi \square\left[\varphi^{\prime}{ }_{M}, \varphi^{\prime}{ }_{Y}\right], \mathrm{r} \square\left[\mathrm{T}_{\mathrm{L}}, 85\right]\right.
$$

\section{Thresholding Using Contourlet Transformation}

Even though CCS method of skin color segmentation is a very efficient method, it fails when the skin tone of the subject lacks chroma. This is due to the fact that skin tones which lack chroma lies in the L region of the centroid hexagon. Since we ignored L region while performing thresholding using CCS algorithm, CCS fails to detect such skin regions. To overcome this shortcoming of CCS, we propose a correcting method based on Contourlet Transformation. In this step we find the contourlet transform of the given image. This transformed image is processed as per the algorithm given in following section. 


\section{FACE DETECTION}

\subsection{Thresholding}

\subsubsection{Thresholding Based on CCS}

Use the CCS can solve the shortage of existing methods based on color analysis, because it can ignore the influence of color and luminance. It only calculates the direction of color and can let the darkly and lightly region comes to one cluster in order to ignore some noise. Using the thresholds selection way as Section II to select thresholds $\varphi_{M}, \varphi_{R}$ and $T_{L}$ to get the threshold curves for thresholding. Other thresholds will keep the initial value and without calculate here. By this way, the binary image can be got as Fig. 4(b). From the result we can see that the white background region (wall), pale color clothing region and dark color clothing region are clustering to black and only the goal region clustering to white. By this way it can ignore many noise regions; especially the excessive bright or dark region and different color region. But because the color of regions near the face color so it is cluster to pre-face region.

\subsubsection{Thresholding based on Contourlet Transform}

The basic steps involved in the proposed CBIR system includes database processing and resizing, creation and normalization of feature database, comparison and image retrieval. Steps of the proposed algorithm are as follows.

1. Decompose each image in the Contourlet domain

2. Compute the standard deviation (SD) of the CT decomposed image on each directional subband. Standard deviation is given as

$\sigma=\sqrt{\frac{1}{M \times N} \sum_{i=1}^{M} \sum_{j=1}^{N}\left(W_{k}(i, j)-\mu_{k}\right)^{2}}$

Where,

$W_{k}=$ coefficient of $\mathrm{k}^{\text {th }} \mathrm{CT}$ decomposed sub-band

$\mu_{k}=$ Mean value of $\mathrm{k}^{\text {th }}$ sub-band

$M \times N=$ Size of the CT decomposed sub-band

The resulting SD vector is

$$
\overline{\boldsymbol{f}}=\left[\sigma_{1}, \sigma_{2}, \sigma_{3}, \ldots \ldots \ldots \sigma_{n}\right]
$$

3. Normalize the SD vector to range [0 1] for every image in the database

4. Apply query image and calculate the feature vector as given in steps 2 to 3 .

5. Calculate the similarity measure Manhattan distance

$$
D_{q i}^{M}=\left|\overline{\boldsymbol{f}}_{\boldsymbol{q}}-\overline{\boldsymbol{f}}_{\boldsymbol{q}}\right|
$$

6. Retrieve all relevant images to query image based on minimum Manhattan distance.

\subsubsection{Correction Using Nonlinear Thresholding}

For denoise the incorrect region, this paper adopt the nonlinear thresholding method to correct the binary image which is thresholded by CCS. Considering the frequency of gray values can more exactly reflect its distributing, so do the nonlinear transform with original image can divide the gray values based luminance information to quantize the values to 2 clusters; lastly do inverse transform to get the binary image. Fig. 4(a) and (c) are the original image and binary image which thresholding by nonlinear thresholding, and the formula as follows: 


$$
f_{\text {Binary }}(x, y)=\frac{\ln \left[1+255 f_{\text {original }}(x, y)\right]}{k \ln [1+255]}
$$

$\mathrm{k}$ is the number of cluster, here let $\mathrm{k}=2$. From Fig. 4(c) it can be seeing that though the nonlinear thresholding, the background of binary image with white color has been clustering to same value as the face region, so it hard to separate the face region with background. But this binary image can conquer some shortage of CCS; for example, the region in Fig. 4. Sometimes, the bright region also can reflect some color and its centroid in the goal region, but in fact it is noise region. For example, the hair region, so use (5) processed image to correct the CCS based method processed the image with "and operation" can get idea result as follows:

$\mathrm{f}_{\text {Final }}(\mathrm{x}, \mathrm{y})=\left(\mathrm{f}_{\mathrm{ccs}}(\mathrm{x}, \mathrm{y})+\mathrm{f}_{\text {contour }}\right) \mathrm{x} \mathrm{f}_{\text {Non linear }}(\mathrm{x}, \mathrm{y})$

Fig. 4(d) is the corrected binary image, it correct the region effetely. Because of the color of region is near the face region color, so it cannot be deleted.

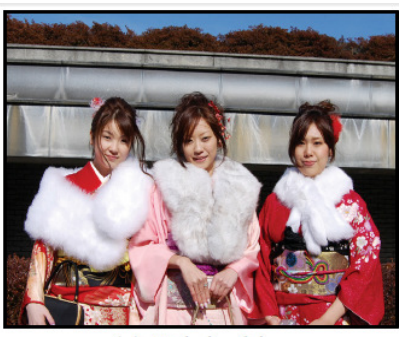

(a) Original image

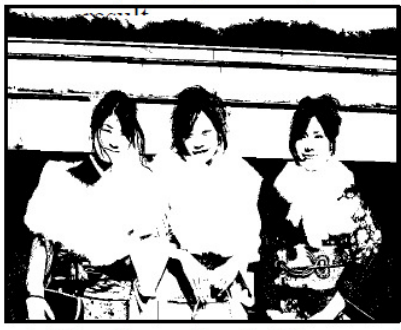

(c) Nonlinear thresholding result

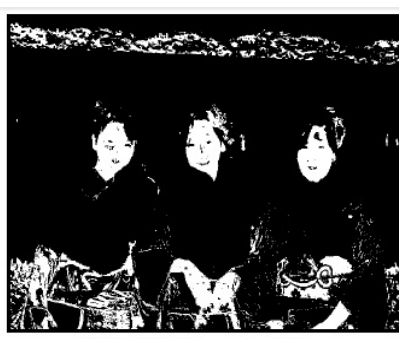

(b) CCS thresholding

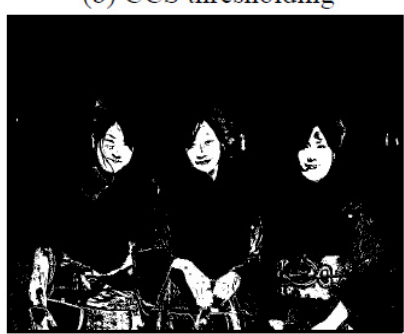

(d) Corrected thresholding

Figure 4. Thresholding Result

\subsection{Pre-face Region Decision}

After get the idea binary image, the white region is the wait-decision region, because it may be include face, hand etc. skin region and other nearly color region (white region on Fig. 5(b)). Here all wait-decision regions are analyzed in a selection process and some of them accepted by aspect ratio and size.

Accepted by aspect ratio:

$\mathrm{C}=4 \pi \mathrm{S} / \mathrm{L}^{2}$

Here $\mathrm{C}$ aspect ratio, $\mathrm{L}$ is the length of boundary. $\mathrm{S}$ is the area of wait-decision region. If $\mathrm{C} \in[1$, 1.7], it will be accepted.

Accepted by size:

After accepted by aspect ratio, then calculate the average area $\mathrm{S}_{\mathrm{AVE}}$ of all wait-decision regions without the largest and smallest regions. If $\mathrm{S}_{\mathrm{FACE}} \in\left(0.8 \mathrm{~S}_{\mathrm{AVE}}, 1.2 \mathrm{~S}_{\mathrm{AVE}}\right)$, it will be accepted. 


\subsection{Face Region Tracking}

Face Tracking is done to indicate the position of detected faces in given image. This is done by drawing a green circle around the detected face.

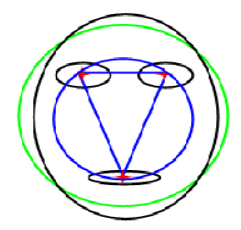

Figure 5. Face tracking model

After face region fixed, use a circle to draw it by follows:

Step1: divide the face region to 9 blocks and thresholding respectively as Fig. 6.

Step2: wipe off noise region by median filter.

Step3: fix eyes and mouth region and then calculate the area centroids of eyes and mouth respectively.

Step4: draw a circum-circle (blue circle of Fig. 6) of triangle which created by the three centroids. Then use its 1.5 times' concentric circle (green circle of Fig. 6) to mark face.

\section{EXPERIMENTAL RESULTS}

Figure 6 shows an example with complex background under outdoor situation, (a) is the original image; (b) is the thresholding result by proposed method; (c) (e)are different results by traditional thresholding methods. Compare those thresholding results; we can easily conclude that the proposed method is better than the others.

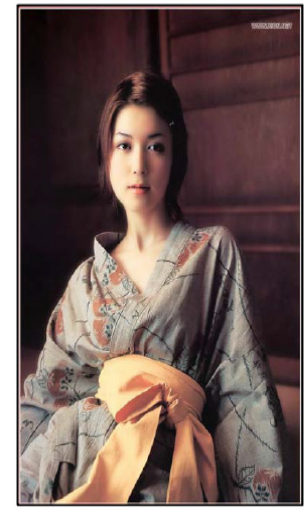

(a) Original image

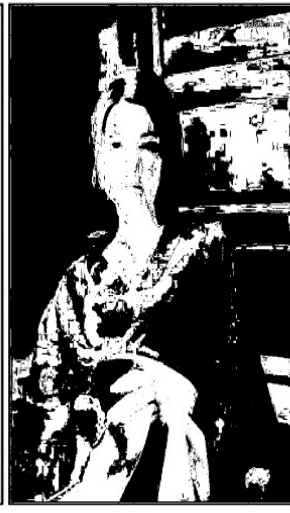

(b) Binary image

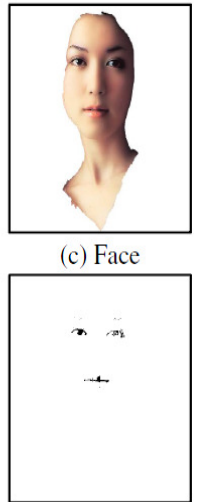

(d) Facial feature

Figure 6. Face region decision of sample image 


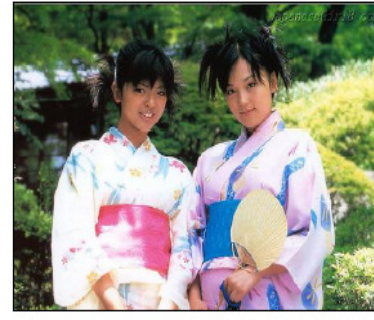

(a) Original image

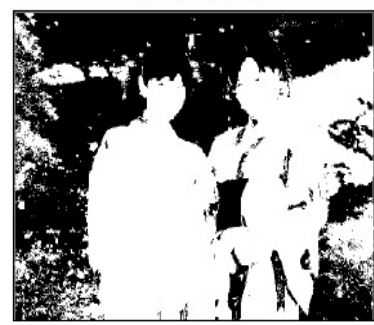

(d) Non-linear thresholding

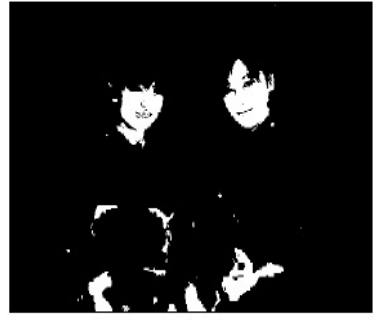

(b) Proposed method

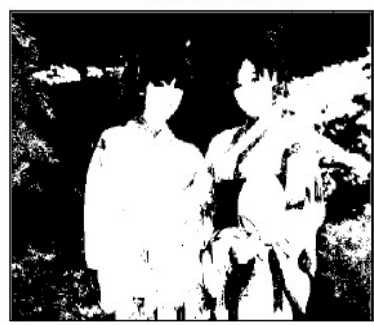

(e) Maximum entropy thresholding

Figure 7. Result Comparison

\section{CONCLUSiON}

As we have seen the face detection locates and segments face regions in cluttered images. It has numerous applications in areas like surveillance and security control systems, content-based image retrieval, video conferencing and intelligent human computer interfaces. Some of the current face-recognition systems assume that faces are isolated in a scene. Through observing experiment result and analysis, the proposed method can detect and track faces under varied conditions effectively. Our detection algorithm takes the color image and applies CCS method and CT method to detect the valid skin regions. Then use the binary image which quantized by nonlinear thresholding to correct the binary image which is thresholded by CCS method and CT method. Finally, using close operation and filter to get the ideal binary image. For tracking faces, use 1.5 times concentric circle of the circum-circle of triangle which created by facial features to mark face. All the experiment result show that the proposed method can get ideal detection and tracking result under complex background, multi-face and color influence. The future works are how to make the CT method more quickly and have better thresholded effect for detecting.

In the future, we need to complete the following items to improve the performance of our method further:

- Overcome the change of pose and view point.

- Use motion analysis for effective prediction.

Integrate the detection and tracking information to make a face model for real-time recognition.

\section{REFERENCES}

[1] Jun Zhang, Qieshi Zhang, and Jinglu Hu: "RGB Color Centroids Segmentation (CCS) for Face Detection" IEEE Transaction on Image Processing, Volume (9), Issue (II), April 2009

[2] T. Gevers, and A.W.M. Smeulders, "Combining Color and Shape Invariant Features for Image Retrieval," IEEE Transactions on Image Processing, Vol. 9, pp. 102-119, Jan. 2000. 
[3] Y. T. Pai, S. J. Ruan, M. C. Shie, and Y.C. Liu, "A Simple and Accurate Color Face Detection Algorithm in Complex Background," 2006 IEEE International Conference on Multimedia and Expo, pp.1545-1548, Jul. 2006.

[4] Q. Zhang, J. Zhang and S. Kamata: "Face Detection Method Based on Color Barycenter Hexagon Model", 2008 International Multi-Conference of Engineers and Computer Scientists, Vol. 1, pp. 655-658, Mar. 2008.

[5] Christopher C Yang, "Content Based Image Retrieval: A comparison between query by example and Image browsing map approaches", Department of Systems Engineering and Engineering Management, The Chinese university of Hong Kong, Hong Kong.

[6] Minakshi Banerjee 1, Malay K. Kundu, Machine Intelligence, "Edge based features for content based image retrieval”, Unit, Indian Statistical Institute, 203, B. T. Road, Kolkata 700 108, India

[7] Ch.Srinivasa Rao , S. Srinivas kumar, B.N.Chatterji “ Content Based Image Retrieval using Contourlet Transform” Research scholar, ECE Dept., JNTUCE, Kakinada, A.P, India. Professor of ECE, JNTUCE, Kakinada, A.P, India. Former Professor, E\&ECE Dept., IIT, Kharagpur, W.B, India.

[8] Efficient CBIR Using Color Histogram Processing by Neetu Sharma., Paresh Rawat and jaikaran Singh. Signal \& Image Processing : An International Journal (SIPIJ) Vol.2, No.1, March 2011

[9] Face Recognition using Simplified Fuzzy Artmap by Antu Annam Thomas and M. Wilscy, Signal\&Image Processing : An International Journal(SIPIJ) Vol.1, No.2, December 2010

[10] Explicit Content Image Detection by Jorge Alberto Marcial Basilio, Gualberto Aguilar Torres Gabriel Sánchez Pérez, Linda Karina Toscano Medina, Héctor Manuel Pérez Meana, Enrique Escamilla Hernadez, Signal \& Image Processing : An International Journal(SIPIJ) Vol.1, No.2,

December 2010

\section{Authors}

Sunish Kumar O S: Mr. Sunish Kumar is currently working as Asst. Professor in an Engineering college at kerala. He did B Tech in Electronics and Communication Engineering from Cochin University of Science and Technology and MBA in HRM \&FINANCE from Kerala University, Trivandrum. Mr. Sunish has authored several paperes in National and International Conferences. His interested areas are Image processing, Digital Signal Processing, Applications of Fuzzy logic in Management etc.

Akas G Kamal: Mr. Akas is currently working as Asst. Professor in an Engineering college at kerala. He did B Tech in Electronics and Communication Engineering from Cochin University of Science and Technology. Now he is pursuing his $\mathrm{M}$ Tech in Communication Engineering from Mahathma Ghandi University Kerala. His interested areas are Image processing, Face recognition, Digital Speech Processing etc.
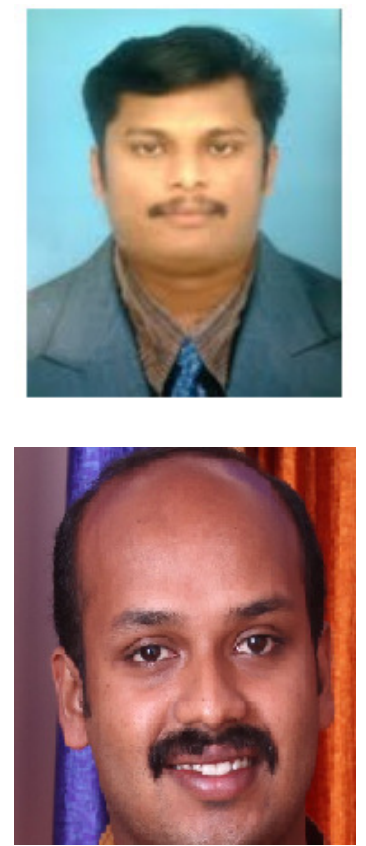